\title{
Establishment of an immortalized mouse dermal papilla cell strain with optimized culture strategy
}

\author{
Haiying Guo ${ }^{1}$, Yizhan Xing ${ }^{1}$, Yiming Zhang ${ }^{2}$, Long He ${ }^{1,3}$, Fang Deng ${ }^{1}$, Xiaogen Ma ${ }^{1}$, Yuhong Li ${ }^{\text {Corresp. }} 1$ \\ 1 Department of Cell Biology, Army Medical University, Chongqing, China \\ 2 Department of Plastic and Cosmetic surgery, Xinqiao Hospital, Army Medical University, Chongqing, China \\ 3 "111" Project Laboratory of Biomechanics and Tissue Repair \& Key Laboratory of Biorheological Science and Technology of Ministry of Education, \\ College of Bioengineering, Chongqing University, Chongqing, China \\ Corresponding Author: Yuhong Li \\ Email address: liyuhongtmmu@hotmail.com
}

Dermal papilla (DP) plays important roles in hair follicle regeneration. Long-term culture of mouse DP cells can provide enough cells for research and application of DP cells. We optimized the culture strategy for DP cells from three dimensions: stepwise dissection, collagen I coating, and optimized culture medium. Based on the optimized culture strategy, we immortalized primary DP cells with SV40 large T antigen, and established several immortalized DP cell strains. By comparing molecular expression and morphologic characteristics with primary DP cells, we found one cell strain named iDP6 was similar with primary DP cells. Further identifications illustrate that iDP6 expresses FGF7 and $\alpha$-SMA, have activity of alkaline phosphatase. During the process of characterization of immortalized DP cell strains, we also found that cells in DP were heterogeneous. We successfully optimized culture strategy for DP cells, and established an immortalized DP cell strain suitable for research and application of DP cells. 
2 Haiying Guo ${ }^{1, \mathrm{a}}$, Yizhan Xing ${ }^{1, \mathrm{a}}$,Yiming Zhang ${ }^{2}$, Long $\mathrm{He}^{1,3}$, Fang Deng ${ }^{1}$, Xiaogen $\mathrm{Ma}^{1}$, Yuhong $\mathrm{Li}^{1, *}$

3 Address:

41 Department of Cell Biology, Army Medical University, Chongqing 400038, China.

52 Department of Plastic and Cosmetic surgery, Xinqiao Hospital, Army Medical University, Chongqing 6 400037, China.

73 "111" Project Laboratory of Biomechanics and Tissue Repair \& Key Laboratory of Biorheological Science

8 and Technology of Ministry of Education, College of Bioengineering, Chongqing University, Chongqing

9400044 , China.

$10{ }^{a}$ These authors contribute equally to the paper

11 "Corresponding author

12 Yuhong Li: Tel: +86 23 68752253, E-mail: liyuhongtmmu@hotmail.com

13 Abstract

14 Dermal papilla (DP) plays important roles in hair follicle regeneration. Long-term culture of mouse DP cells 15 can provide enough cells for research and application of DP cells. We optimized the culture strategy for DP 16 cells from three dimensions: stepwise dissection, collagen I coating, and optimized culture medium. Based on

17 the optimized culture strategy, we immortalized primary DP cells with SV40 large T antigen, and established

18 several immortalized DP cell strains. By comparing molecular expression and morphologic characteristics with

19 primary DP cells, we found one cell strain named iDP6 was similar with primary DP cells. Further

20 identifications illustrate that iDP6 expresses FGF7 and $\alpha$-SMA, have activity of alkaline phosphatase. During

21 the process of characterization of immortalized DP cell strains, we also found that cells in DP were

22 heterogeneous. We successfully optimized culture strategy for DP cells, and established an immortalized DP

23 cell strain suitable for research and application of DP cells. 


\section{Introduction}

Hair follicle has the characteristic of periodically growth, which provides nice model for the research of tissue regeneration. Dermal papilla (DP) cells have contact with hair follicle stem cells regularly and may play important roles in the regeneration of hair follicle (Su et al. 2017; Woo et al. 2017). The signals from DP may regulate the regeneration of hair follicles and melanocyte (Guo et al. 2016; Li et al. 2013). Dissociated human DP cells induce hair follicle neogenesis in grafted dermal-epidermal composites (Thangapazham et al. 2014). The limitation for DP research lies in the difficulty for culture of DP cells (Morgan 2014). As so far, the human intact dermal papilla transcriptional signature can be partially restored by growth of papilla cells in 3D spheroid cultures (Topouzi et al. 2017). When the culture environment was changed into 2D environment, very rapid and profound molecular signature changes were discovered (Higgins et al. 2013; Lin et al. 2016). The isolation method of DP by surgical microdissection has been established in mouse vibrissae follicles and in human hair follicles (Gledhill et al. 2013), but the isolated DP cells can not be long-term cultured. Since the isolation of primary DP cells is time-consuming and has limited population doubling. There are also several inter-individual and intra-individual variations. It is necessary to establish stable DP cell lines to investigate hair biology. Immortalized DP cell lines of human have been established, and had hair growth promoting effects (Shin et al. 2011; Won et al. 2010). In rodent animal models, immortalized rat DP cells have already been obtained (Kang et al. 2015). However, an effective immortalized mouse DP cell line is to be constructed. The goals of this project are optimize the isolation and culture condition of DP from mouse skin and establish an immortalized DP cell line for future research.

\section{Materials and Methods}

\subsection{Isolation and culture of DP cells}

C57BL/6 mice were obtained from and housed in the laboratory animal center of the Army Medical University, Chongqing, China. All the animal-related procedures were conducted in strict accordance with the approved institutional animal care and maintenance protocols. All experimental protocols were approved by the Laboratory Animal Welfare and Ethics Committee of the Army Medical University. Permission number for producing animals: SCXK-PLA-20120011. Permission number for using animals: SYXK-PLA-20120031.

A 9-day old C57BL/6 mouse was sacrificed according to standard protocol. The vibrissa pads were cut off bilaterally with an iris scissor in a $100-\mathrm{mm}$ plate. Vibrissa pads were rinsed with PBS, and then hair follicles were dissected together with their connective tissue sheath using $27 \mathrm{G}$ syringe needles under dissecting microscope. The dissected hair follicles were rinsed with PBS and incubated with $0.25 \%$ dispase for 20 minutes at room temperature.

Dissected hair follicles were transferred into a new 100-mm plate and thoroughly washed with PBS. A horizontal cut directly above dermal papilla was made. After that, dermal papilla was dissected out of dermal sheath using $27 \mathrm{G}$ syringe needles under dissecting microscope. Then the dissected DP tissues were transferred into a $10 \mu \mathrm{g} / \mathrm{cm}^{2}$ collagen I coated 24-well plate. DP media were added after 30 minutes incubation in $37{ }^{\circ} \mathrm{C}$. DP cells presented at about 3 days later. Cells reach confluence after 2 weeks and were passaged onto collagen I coated plates. DP medium should include $\alpha$-MEM(Gibco, USA), 10\% FBS (Gibco, USA), $1 \times$ sodium pyruvate (Gibco, USA), $1 \times$ non-essential amino acid (Gibco, USA), $1 \times$ penicillin-streptomycin, $10 \mathrm{ng} / \mathrm{ml}$ bFGF (peprotech, USA). During the optimization process, the classical DP medium was used as control. The 
63 control medium is consisted of DMEM (Gibco, USA), 10\% FBS (Gibco, USA), $1 \times$ penicillin-streptomycin.

64 Another control medium was the classical DP medium with the addition of $10 \mathrm{ng} / \mathrm{ml} \mathrm{bFGF}$ (peprotech, USA).

\subsection{Establishment of immortalized DP cell line}

Retrovirus with SV40 large T antigen which was flanked with FRT sites was prepared as formerly reported (Yang et al. 2012). Primary DP cells were plated in a $60 \mathrm{~mm}$-dish at $50 \%$ confluency in the morning. After attachment, polybrene (final concentration $10 \mu \mathrm{g} / \mathrm{mL}$ ) and retrovirus $\left(3.0 \times 10^{7} \mathrm{TU}\right.$ ) were added together. The second day, the supernatant was aspirated out of dish, and new DP medium was refilled. At the same time, hygromycin was added at a final concentration of $200 \mu \mathrm{g} / \mathrm{mL}$. The culture medium was changed every two days until all the cells in control group died.

Antibiotic-selected DP cells were diluted with DP medium to 1 to 2 cells per $100 \mu \mathrm{L}$, and $100 \mu \mathrm{L}$ diluted cells were added into every well of 96-well plates. Wells with only one cell were labeled and were monitored every 2 days. Cells in the labeled wells were passaged when the cell number of clones reached 20 or more.

\subsection{RT-PCR}

Total RNA of immortalized DP cells were extracted with Eastep ${ }^{\mathrm{TM}}$ super total RNA extraction kit (Promega, China) according to manufacturer's protocol. Complementary DNA was synthesized from RNA using Rever Tre Ace cDNA synthesis Kit (TOYOBA, Japan) according to the manufacturer's protocol. Several gene expressions were determined by PCR machine (Bio-Rad, USA) with the synthesized cDNA as template. The primers used were shown in Table 1. PCR mastermix (Novoprotein, China) were used when amplifying. The reannealing temperatures $(\mathrm{Tm})$ and product size for the primers were also shown in Table 1.

Table 1 The information of primers in RT-PCR experiment

\subsection{Immunocytochemistry staining}

Cover slides were placed on a 24-well plate, and cells were plated on cover slides. Twenty-four hours later, cover slides were rinsed with PBS and fixed with acetone. Then, the cover slides were rinsed with PBS and incubated with 5\% goat serum in PBS at room temperature for 1 hour. After that, slides were incubated with a rabbit anti-FGF7 antibody (1:100, Boster, China) or a rabbit anti- $\alpha$-SMA antibody (1:200, Bioss, China) at $4{ }^{\circ} \mathrm{C}$ overnight and subsequently with appropriate secondary antibodies (1:500, ZSGB-bio, China). The slides were counterstained with DAPI (1:10000, Beyotime, China) for 10 minutes. At last, the cover slides were moved to microscope slides, mounted with antifade mounting medium (Beyotime, China), and observed under fluorescent microscope. The immunostaining experiments were repeated for 3 times.

\subsection{Alkaline phosphatase staining}

Cover slides were placed on a 24-well plate, and cells were plated on cover slides. Twenty-four hours later, cover slides were rinsed with PBS and fixed with in situ fixation solution (Beyotime, China) for 10 minutes. Then the cover slides were rinsed with PBS for 5 times. Fresh made NBT/BCIP staining buffer (Beyotime, China) or BM purple (Roche, USA) were added into the wells. The plate was covered with aluminium foil in 
97

the dark. Color change was monitored every 15 minutes to avoid non-specific staining. After the colour change appeared, the staining solution was aspirated out and the cells were washed twice with $1 \times$ PBS. At last, the cover slides were dehydrated, cleared, moved to microscope slides, mounted with permount (ZSGB-bio, China), and observed under microscope. The AP staining experiments were performed twice.

\subsection{Detection of immortalization}

Primary DP cells and iDP6 cells were cultured. The iDP6 cells were treated with AdGFP (adenovirus with the ability to express GFP protein), AdFlip (adenovirus with the ability to express flip recombinase, which can interact with FRT thus remove the expression of SV40) or PBS. Forty-eight hours later, cells were collected and total proteins were extracted with RIPA lysis buffer (Beyotime, China). Then, total proteins were loaded to 1\% SDS-PAGE gel (Beyotime, China) and transmitted to PVDF membrane (Bio-Rad, USA). The PVDF membrane were incubated with anti-SV40 (1:1000, Santa Cruz, USA) and anti-GAPDH (1:500, ZSGB-bio, China) antibodies. HRP labelled secondary antibodies were used, and the results were observed under ChemiDoc ${ }^{\mathrm{TM}}$ Touch Imaging System (Bio-Rad, USA). The experiment on reversing immortalization was performed twice.

\section{Results}

\subsection{DP cells can be long-term cultured with the optimized strategy}

We optimized the culture strategy for DP cells from three dimensions, plate coating, dissecting method, and culture media (Figure 1). The optimized dissecting method worked well in obtaining primary DP cells. DP cells grew better on plate coated with collagen I than on uncoated plate. The morphology of DP cells did not have any significant difference between classical DP culture medium (DMEM with 10\% FBS) and classical DP culture medium with the addition of bFGF (data not shown). Compared with classical DP culture medium, primary DP cells grew better in the optimized culture medium (Figure 2A-2D). The morphology of passaged DP cells was much more resemble in primary DP cells in the optimized culture medium. The cultured DP cells still had the characteristics of agglutinative growth in the optimized culture medium, but not in the control medium (Figure 2E-2H).

\subsection{DP cells are heterogeneous}

Primary DP cells were immortalized by SV40 system. DP cells before antibiotic-selection were named with 0\#. After antibiotic-selection, DP cell strains were selected by infinite dilution method. Not every single cell grew to clone at last. Cell strains were named with the time sequence when they grew to clone beginning with just one single cell. Totally 19 cell strains survived at last, named with iDP1 to iDP19 (1\# to 19\#). The morphologic characteristics of the selected cell strains were different from each other (Figure 3). Some cells still look like fibroblast, whereas some cells changed into epithelial-like cells (Figure 3G). iDP6 still had the characteristic of agglutinative growth, while others lost this characteristic. Specially, iDP10 grew clonally, which implied that the cell line was more primitive. For these cell strains, the expression patterns of the markers for DP cells were also determined by RT-PCR, including FGF7, BMP6, Sox2, Tbx18, Sostdc, $\alpha$-SMA and noggin (Figure 4). All these data indicate that the cell strains were totally different from each other. Since each cell line grew from one single DP cell, the DP cells from one DP tissue were heterogeneous. 


\section{3 iDP6 keeps the molecular characteristics of primary DP cells}

Taken morphology and mRNA expression characteristics together, iDP6 is the one that most similar to the primary DP cells. To determine whether iDP6 can be used in DP research, the activity of alkaline phosphatase was determined by AP staining, and the expression of FGF7 and $\alpha$-SMA were determined by immunocytochemistry as well. At protein level, just like in situ, some iDP6 cells still had high AP activity (Figure 5A-5F). FGF7 was expressed in the cytoplasm of all iDP6 cells (Figure 5G-5L). $\alpha$-SMA was expressed in both the cytoplasm and the nucleus of all iDP6 cells (Figure 5M-5R). Although the AP activity in iDP6 was lower than primary DP cells, the expression patterns of FGF7 and $\alpha$-SMA was similar to primary DP cells.

\subsection{The immortal process of DP cells is reversible}

At first, the expression of SV40 were determined to make sure that iDP6 were immortalized. Western blot showed that iDP6 cells expressed SV40, while primary DP cells did not express SV40 (Figure 6A). Then, AdFlip was used to remove the expression of SV40 in iDP6 cells. AdGFP and PBS were used as control. Results showed that compared with control groups, the expression of SV40 decreased at 48 hours after being treated with AdFlip (Figure 6B). These results demonstrated that iDP6 was successfully immortalized and the immortal process was reversible.

\section{Discussion}

Primary cell culture needs to simulate the in vivo environment of the cells. In anagen, DP cells reside in the center of hair bulb. They are circumstanced with a single layer of dermal cells. Usually, DP cells periodically interact with epithelial cells outside of the single layer of dermal cells. In telogen, DP tissue is a little far from hair follicle stem cells (HFSCs). In anagen onset, it begins to move close to HFSCs, and keeps interaction with HFSCs during early anagen. In the late anagen, it begins to move away from HFSCs. In catagen, it keeps away from HFSCs. Thus, the environment for DP cells in vivo varies with hair cycle (Bassino et al. 2015). In addition, exogenous connective tissue may also impact the function of DP cells (Zhang et al. 2014b). To exclude contamination from other mesenchymal cells, epithelial cells, and adipose tissue, the use of microdissection techniques is preferred (Zhang et al. 2014a). To culture DP cells in vitro, all the conditions should be taken into consideration. As the main component of connective tissue, collagen is mostly secreted by fibroblast. Collagen is widely used in tissue engineering and cell culture. We coated the plate with collagen, and found it was good for the growth of DP cells. DP is relatively independent in the anagen hair follicle. However, it is too small to be isolated quickly. So we used a stepwise method to isolate DP. DP cells grew from the isolated DP. The most important condition for cell culture is the culture medium. Since the classical DMEM with 10\% FBS can not long-term culture mouse DP cells, we seek to find culture medium for special fibroblast. We found that a mesenchymal stem cell culture medium $\alpha$-MEM worked well. Additionally, bFGF is a critical component of human embryonic stem cell culture medium. In conjunction with BMP4, bFGF promotes differentiation of stem cells to mesodermal lineages (Yuan et al. 2013). DP originates from mesodermal, so we added bFGF to the medium. However, since the classical medium and the classical medium with the addition of bFGF did not have significant difference on the culture of primary DP, the main effective additions in the optimized medium maybe sodium pyruvate and non-essential amino acids. Based on these data, the optimized 
170

171

172

173

174

175

176

177

178

179

180

181

182

183

184

185

186

187

188

189

190

strategy works well in isolating and long-term culture of DP cells. We are the first to use this strategy to culture DP cells.

There are several molecules reported to be expressed in DP cells, including Sox 2 , Tbx18, Sostdc, $\alpha$-SMA and noggin (Weber \& Chuong 2013). To characterize immortalized DP cells, all the markers were tested. Recently, we found that two secretive proteins, FGF7 and BMP6 were also expressed in DP cells in vivo. FGF signaling was reported to regulate the size of dermal papilla (Yue et al. 2012), and BMP7 was reported to attenuate fibroblast-like differentiation of DP cells (Bin et al. 2013). Thus the expressions of FGF7 and BMP6 are also tested. Both the expressions of markers and morphology indicate that the immortalized DP cell strains are heterogeneous and iDP6 is a good cell strain to represent primary DP cells. It is reported that human DP cells have stem cell-like phenotypes (Kiratipaiboon et al. 2016), neural crest stem cell-like cells were also isolated from rat vibrissa DP (Li et al. 2014), dermal stem cells also lies in mouse dermal sheath (Rahmani et al. 2014). So it is reasonable that DP cells are heterogeneous. But exactly how many kinds of cells are in DP remain to be discovered (Yang et al. 2017). Single cell assay technologies may help.

\section{Conclusions}

From the results of present study, it can be concluded that we optimized the dissection and culture of mouse DP cells from three dimensions: stepwised dissction, collagen I coated plate and $\alpha$-MEM based culture medium. Based on the optimized strategies, we successfully immortalized the cultured primary DP cells with addition of SV40 large T antigen. We successfully selected several cell strains, characterized them, and found iDP6 cell strain similar to primary DP cells. In addition, the SV40 large T antigen in iDP6 can be removed by the addition of AdFlip. In a word, we establised an immortalized DP cell strain that can be used in future research.

\section{Acknowledgements}

We thank Tong-chuan He in Chicago University for providing the SV40 large T antigen associated plasmids. We thank Ke Yang in Chongqing Medical University for helping in the immortalization experiments. We thank Claire Higgins and other reviewers for constructive suggestions on the revision of the manuscript.

\section{References}

Bassino E, Gasparri F, Giannini V, and Munaron L. 2015. Paracrine crosstalk between human hair follicle dermal papilla cells and microvascular endothelial cells. Exp Dermatol 24:388-390.

Bin S, Li HD, Xu YB, Qi SH, Li TZ, Liu XS, Tang JM, and Xie JL. 2013. BMP-7 attenuates TGF-beta1-induced fibroblast-like differentiation of rat dermal papilla cells. Wound Repair Regen 21:275-281.

Gledhill K, Gardner A, and Jahoda CA. 2013. Isolation and establishment of hair follicle dermal papilla cell cultures. Methods Mol Biol 989:285-292.

Guo H, Xing Y, Liu Y, Luo Y, Deng F, Yang T, Yang K, and Li Y. 2016. Wnt/ $\beta$ catenin signaling pathway activates melanocyte stem cells in vitro and in vivo. J Dermatol Sci 83:45-51. 
Higgins CA, Chen JC, Cerise JE, Jahoda CA, and Christiano AM. 2013. Microenvironmental reprogramming by three-dimensional culture enables dermal papilla cells to induce de novo human hair-follicle growth. Proc Natl Acad Sci U S A 110:19679-19688.

Kang JI, Kim SC, Kim MK, Boo HJ, Kim EJ, Im GJ, Kim YH, Hyun JW, Kang JH, Koh YS, Park DB, Yoo ES, and Kang HK. 2015. Effects of dihydrotestosterone on rat dermal papilla cells in vitro. EurJ Pharmacol 757:74-83.

Kiratipaiboon C, Tengamnuay P, and Chanvorachote P. 2016. Ciprofloxacin Improves the Stemness of Human Dermal Papilla Cells. Stem Cells Int 2016:5831276.

Li M, Liu JY, Wang S, Xu H, Cui L, Lv S, Xu J, Liu S, Chi G, and Li Y. 2014. Multipotent neural crest stem cell-like cells from rat vibrissa dermal papilla induce neuronal differentiation of PC12 cells. Biomed Res Int 2014:186239.

Li YH, Zhang K, Yang K, Ye JX, Xing YZ, Guo HY, Deng F, Lian XH, and Yang T. 2013. Adenovirus-mediated Wnt10b overexpression induces hair follicle regeneration. J Invest Dermatol 133:42-48.

Lin B, Miao Y, Wang J, Fan Z, Du L, Su Y, Liu B, Hu Z, and Xing M. 2016. Surface Tension Guided Hanging-Drop: Producing Controllable 3D Spheroid of High-Passaged Human Dermal Papilla Cells and Forming Inductive Microtissues for Hair-Follicle Regeneration. ACS Appl Mater Interfaces 8:5906-5916.

Morgan BA. 2014. The dermal papilla: an instructive niche for epithelial stem and progenitor cells in development and regeneration of the hair follicle. Cold Spring Harb Perspect Med 4:a015180.

Rahmani W, Abbasi S, Hagner A, Raharjo E, Kumar R, Hotta A, Magness S, Metzger D, and Biernaskie J. 2014. Hair follicle dermal stem cells regenerate the dermal sheath, repopulate the dermal papilla, and modulate hair type. Dev Cell 31:543-558.

Shin SH, Park SY, Kim MK, Kim JC, and Sung YK. 2011. Establishment and characterization of an immortalized human dermal papilla cell line. BMB Rep 44:512-516.

Su YS, Fan ZX, Xiao SE, Lin BJ, Miao Y, Hu ZQ, and Liu H. 2017. Icariin promotes mouse hair follicle growth by increasing insulin-like growth factor 1 expression in dermal papillary cells. Clin Exp Dermatol 42:287294.

Thangapazham RL, Klover P, Wang JA, Zheng Y, Devine A, Li S, Sperling L, Cotsarelis G, and Darling TN. 2014. Dissociated human dermal papilla cells induce hair follicle neogenesis in grafted dermal-epidermal composites. J Invest Dermatol 134:538-540.

Topouzi H, Logan NJ, Williams G, and Higgins CA. 2017. Methods for the isolation and 3D culture of dermal papilla cells from human hair follicles. Exp Dermatol 26:491-496. 
Weber EL, and Chuong CM. 2013. Environmental reprogramming and molecular profiling in reconstitution of human hair follicles. Proc Natl Acad Sci U S A 110:19658-19659.

Won $\mathrm{CH}$, Choi SJ, Kwon OS, Park WS, Kang YJ, Yoo HG, Chung JH, Cho KH, Eun $\mathrm{HC}$, and Kim KH. 2010. The establishment and characterization of immortalized human dermal papilla cells and their hair growth promoting effects. J Dermatol Sci 60:196-198.

Woo H, Lee S, Kim S, Park D, and Jung E. 2017. Effect of sinapic acid on hair growth promoting in human hair follicle dermal papilla cells via Akt activation. Arch Dermatol Res.

Yang H, Adam RC, Ge Y, Hua ZL, and Fuchs E. 2017. Epithelial-Mesenchymal Micro-niches Govern Stem Cell Lineage Choices. Cell.

Yang K, Chen J, Jiang W, Huang E, Cui J, Kim SH, Hu N, Liu H, Zhang W, Li R, Chen X, Kong Y, Zhang J, Wang J, Wang L, Shen J, Luu HH, Haydon RC, Lian X, Yang T, and He TC. 2012. Conditional immortalization establishes a repertoire of mouse melanocyte progenitors with distinct melanogenic differentiation potential. J Invest Dermatol 132:24792483.

Yuan S, Pan Q, Fu CJ, and Bi Z. 2013. Effect of growth factors (BMP-4/7 \& bFGF) on proliferation $\&$ osteogenic differentiation of bone marrow stromal cells. Indian J Med Res 138:104-110.

Yue Z, Jiang TX, Wu P, Widelitz RB, and Chuong CM. 2012. Sprouty/FGF signaling regulates the proximal-distal feather morphology and the size of dermal papillae. Dev Biol 372:45-54.

Zhang P, Kling RE, Ravuri SK, Kokai LE, Rubin JP, Chai JK, and Marra KG. 2014a. A review of adipocyte lineage cells and dermal papilla cells in hair follicle regeneration. J Tissue Eng 5:2041731414556850.

Zhang P, Ravuri SK, Wang J, Marra KG, Kling RE, and Chai J. 2014b. Exogenous connective tissue growth factor preserves the hair-inductive ability of human dermal papilla cells. Int J Cosmet Sci 36:442-450. 


\section{Table 1 (on next page)}

The information of primers in RT-PCR experiment 


\begin{tabular}{|c|c|c|c|}
\hline Primers & Sequence & $\operatorname{Tm}\left({ }^{\circ} \mathrm{C}\right)$ & Product size \\
\hline Noggin- $f$ & 5' AGCACCCAGCGACAACCT 3' & 61 & $343 \mathrm{bp}$ \\
\hline Noggin-r & 5' CAGCCACATCTGTAACTTCCTC 3' & & \\
\hline Tbx18-f & 5' GCTGCTAACCAGACCCAC 3' & 58 & $537 \mathrm{bp}$ \\
\hline Tbx18-r & 5' GTCCATGTCGCCAATACTC 3' & & \\
\hline Bmp6-f & 5' TGCCTTAAACCACGAACAA 3' & 58 & $345 \mathrm{bp}$ \\
\hline Bmp6-r & 5' GCTGGGAATGGAACCTGAA 3' & & \\
\hline Fgf7-f & 5' AGCGGAGGGGAAATGTTCG 3' & 61 & $238 \mathrm{bp}$ \\
\hline Fgf7-r & 5' TCCAGCCTTTCTTGGTTACTGAGA 3' & & \\
\hline Sostdc1-f & 5' CCCCCATCCCAGTCATTTCTT 3' & 58 & $308 \mathrm{bp}$ \\
\hline Sostdc1-r & 5' CAGGGGGATAATTTCACACTGAGA 3' & & \\
\hline Sox2-f & 5' AAAACCGTGATGCCGACTA 3' & 58 & $431 \mathrm{bp}$ \\
\hline Sox2-r & 5' ATCCGAATAAACTCCTTCCTTG 3' & & \\
\hline SMA-f & 5' AGGGAGTAATGGTTGGAATGG 3' & 58 & $351 \mathrm{bp}$ \\
\hline SMA-r & 5' CATCTCCAGAGTCCAGCACAA 3' & & \\
\hline Gapdh-f & 5' ACCACAGTCCATGCCATCAC 3' & 52 & $450 \mathrm{bp}$ \\
\hline Gapdh-r & 5' TCCACCACCCTGTTGCTGTA 3' & & \\
\hline
\end{tabular}

3

4 


\section{Figure 1}

Optimized strategy for the isolation and culture of DP cells

At first, the whole skin of vibrissa area was cut, then the DP tissue was separated from the skin together with vibrissa pad, and then the DP tissue was collected after dispase digestion. After that, the collected DP tissue was cultured with our optimized culture medium in collagen I-coated plate. 


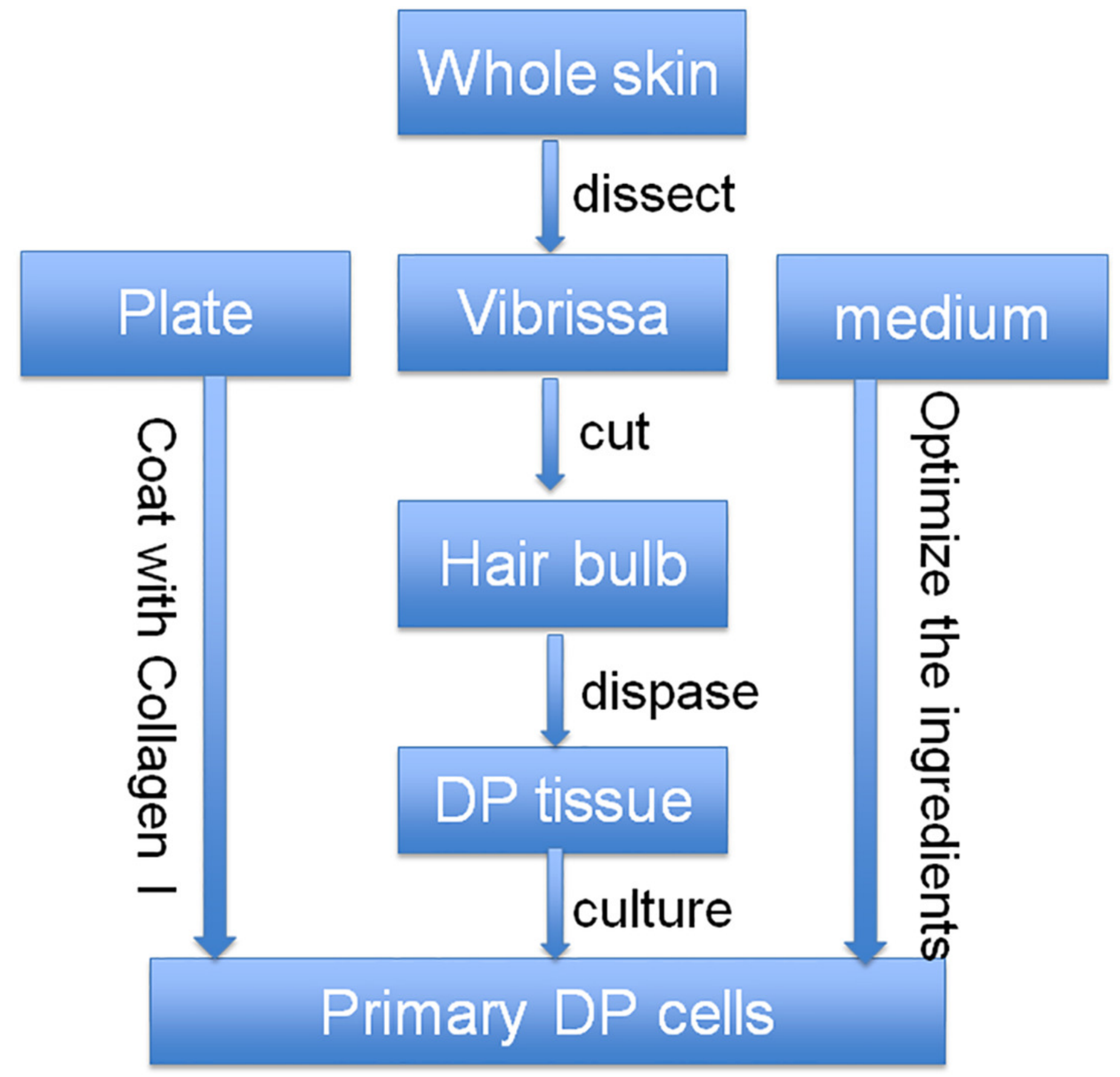


Figure 2

Optimization of culture media for DP cells

Cells in left panel $(A, C, E, G)$ are cultured in DMEM culture medium with $10 \%$ FBS, cells in right panel $(B, D, F, H)$ are cultured in optimized culture medium. $A$ to $D$ are primary DP cells. $A$ and $B$ are 2 days after culture, $C$ and $D$ are 4 days after culture. $E$ to $H$ are DP cells after 1 generation of passage. $\mathrm{E}$ and $\mathrm{F}$ are 2 days after passage, $\mathrm{G}$ and $\mathrm{H}$ are 4 days after passage (100X). Scale bar $=100 \mu \mathrm{m}$. 

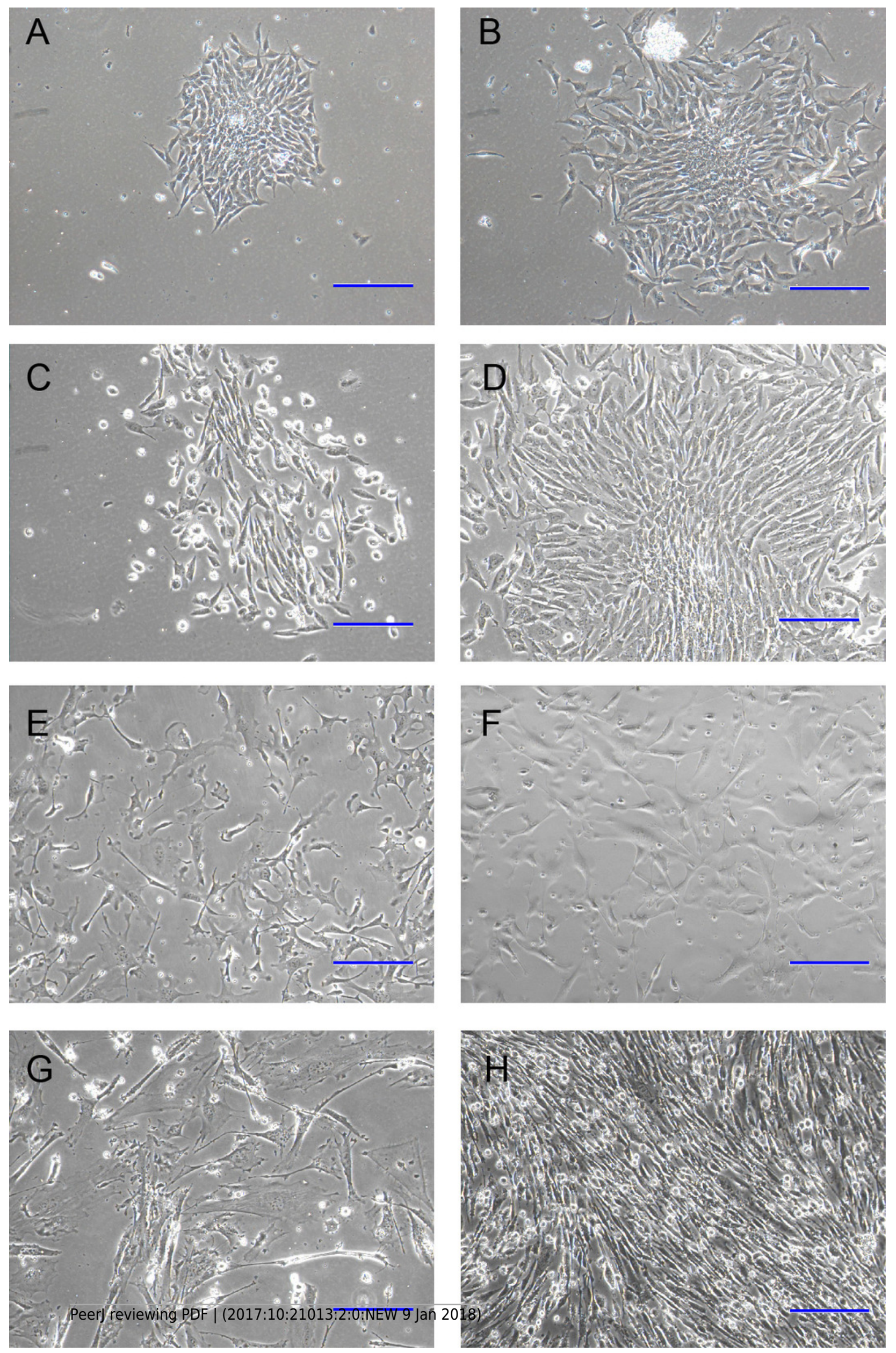
Figure 3

Morphology of immortalized cell strains

A to $L$ represent cell strains named with $0 \#, 3 \#, 4 \#, 5 \#, 6 \#, 9 \#, 10 \#, 11 \#, 12 \#, 14 \#, 17 \#$, 19\#. Scale bar $=100 \mu \mathrm{m}$.
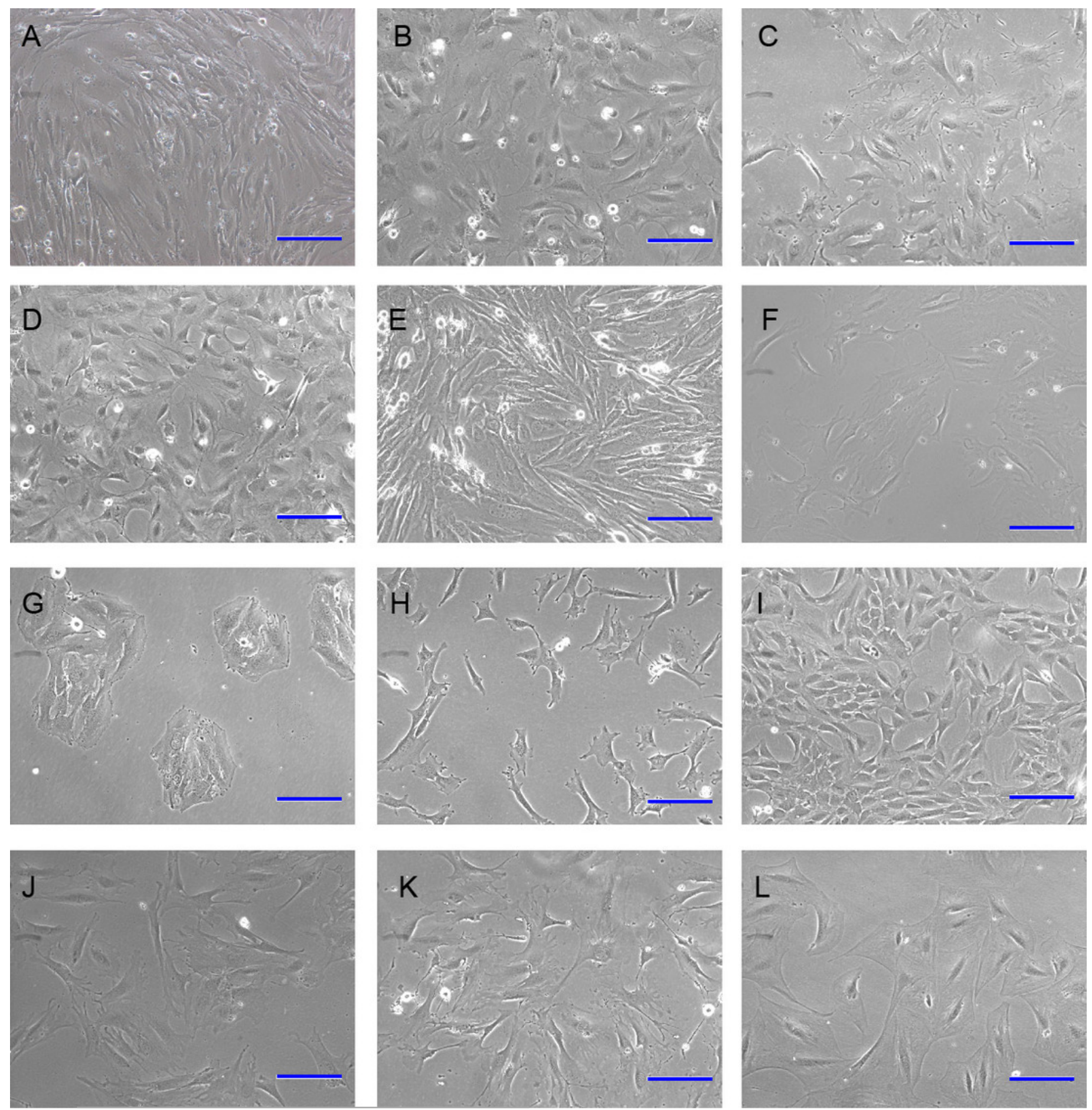


\section{Figure 4}

Expression pattern of the iDP6

The expression of several known DP markers were determined by RT-PCR. GAPDH was used as internal control. Each lane represents a DP cell strain. pDP: primary DP cells.

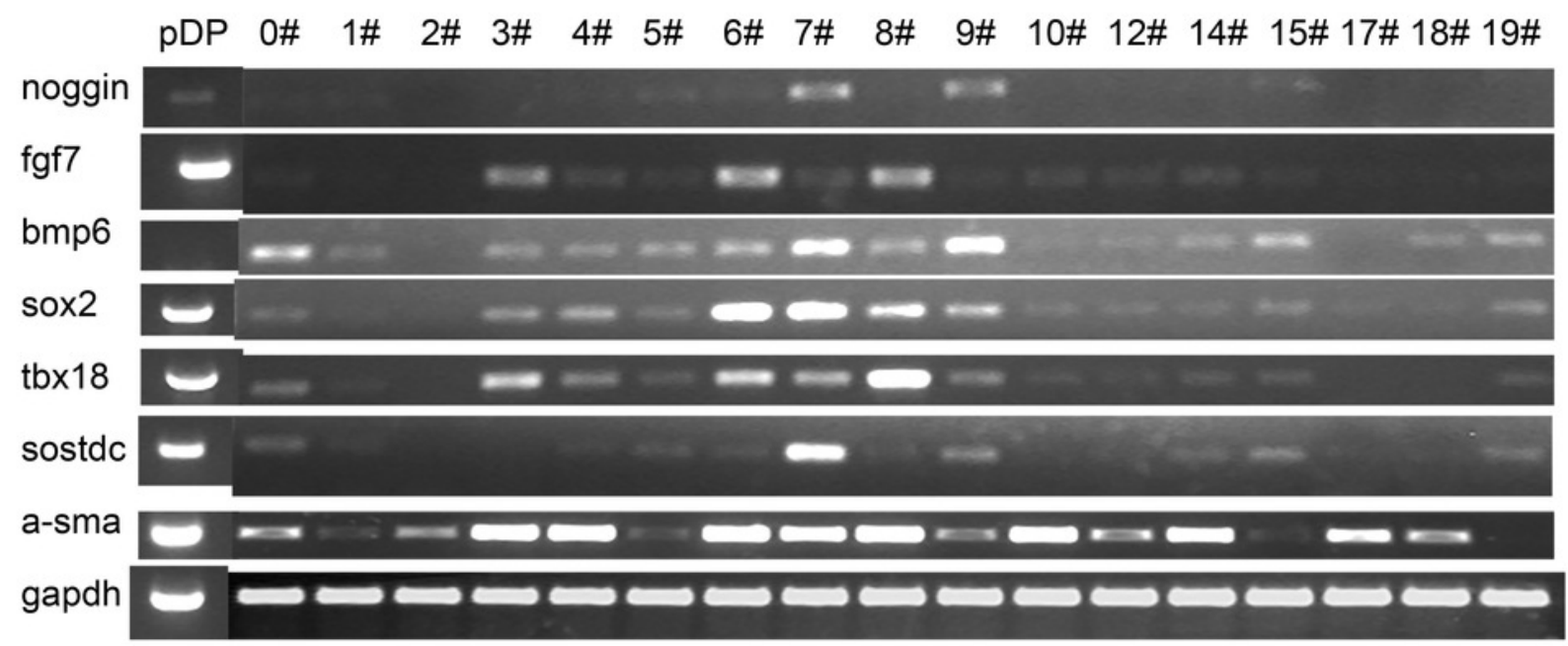




\section{Figure 5}

Characterization of the iDP6

A-C, G-I, M-N Characterization of iDP6. D-F, J-L, P-R Characterization of primary DP cells. A-F Alkaline phosphatase staining. G-R Immunocytochemistry, red color denotes positive expression, blue color denotes the counterstaining of DAPI. G-R The right panels are the merge of left two panels. Arrowheads denote the positive expression. Scales bars for $A$ and $D$ are $100 \mu \mathrm{m}$. Scale bars for $B, E$, and $M-R$ are $50 \mu \mathrm{m}$. Scale bars for $C, F$ and G-L are $25 \mu \mathrm{m}$. 

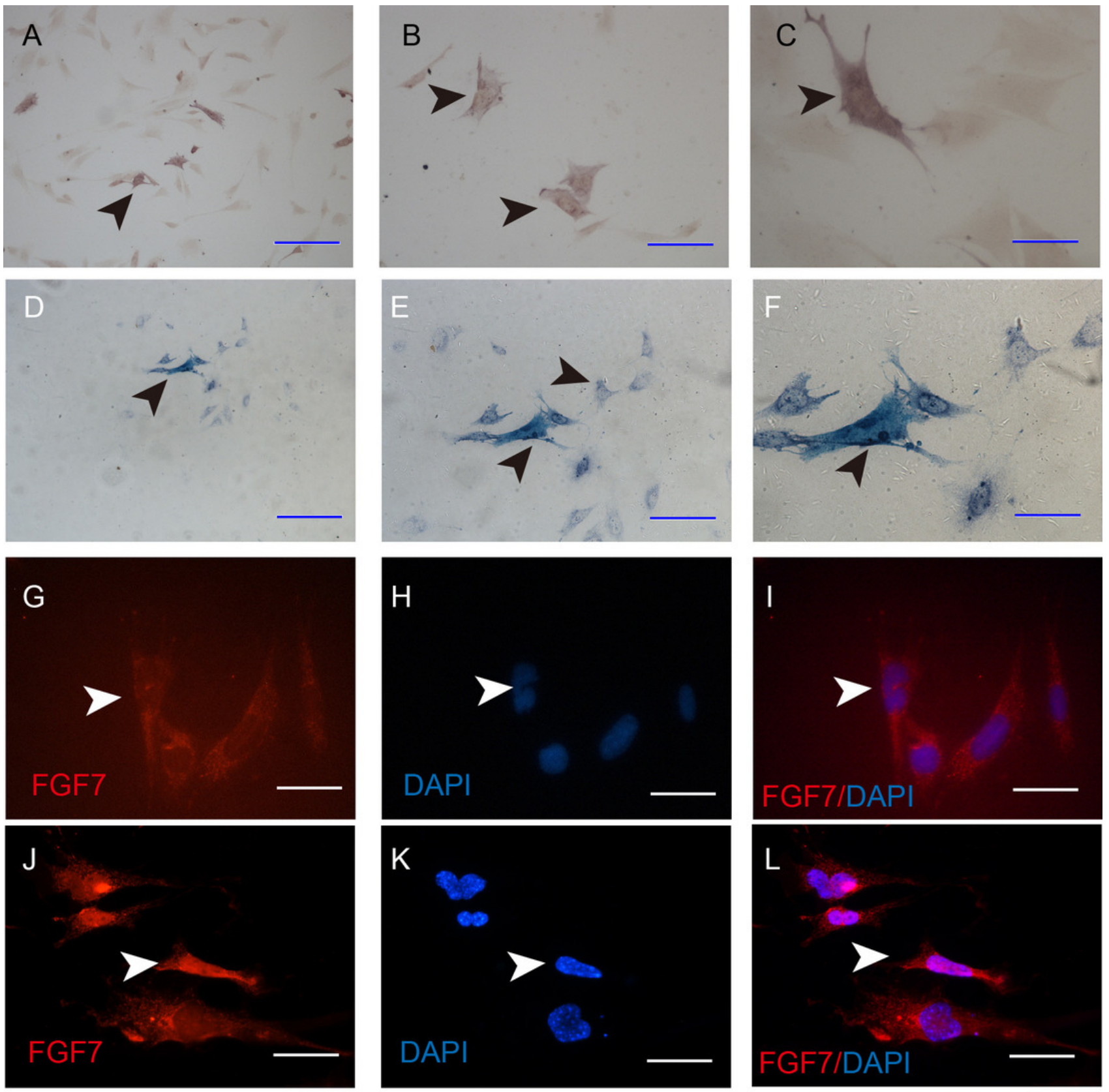

K
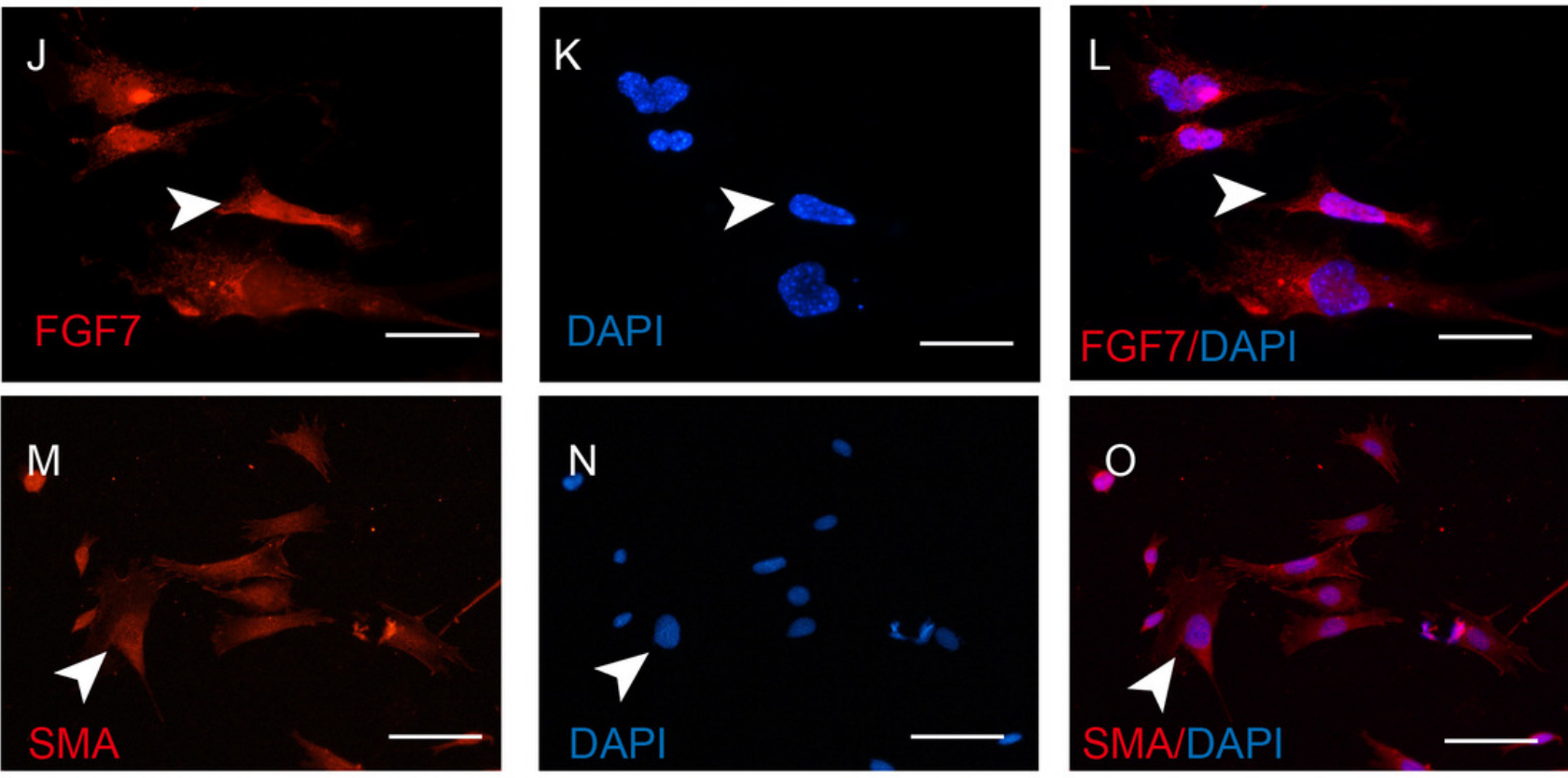

DAPI

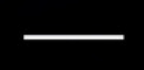

FGF7/DAPI

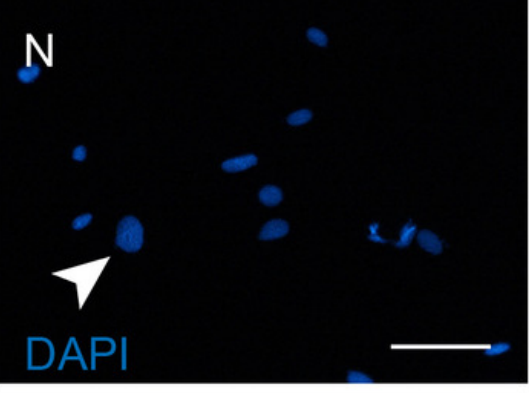

O

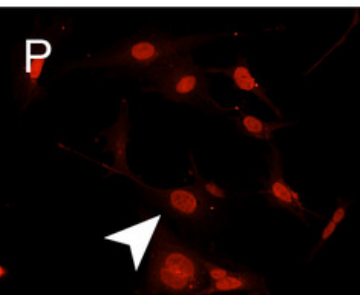

Q

\section{SMA/DAPI}

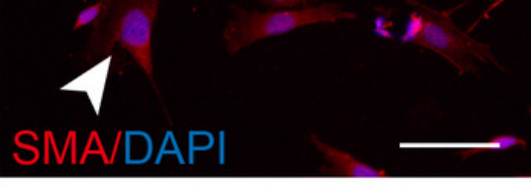




\section{Figure 6}

Reversible immortalization of DP cells

The expressions of SV40 large T antigen were determined by western blot. DP: primary dermal papilla cells. iDP6: immortalized DP cells \#6. AdGFP, AdFlip, PBS: iDP6 cells treated with AdGFP, AdFlip or PBS. Proteins were collected at 48 hours after treatment.

A

iDP6 DP

SV40

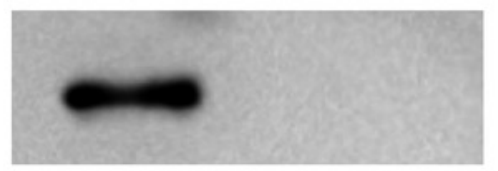

GAPDH
B

AdGFP AdFlip PBS

SV40

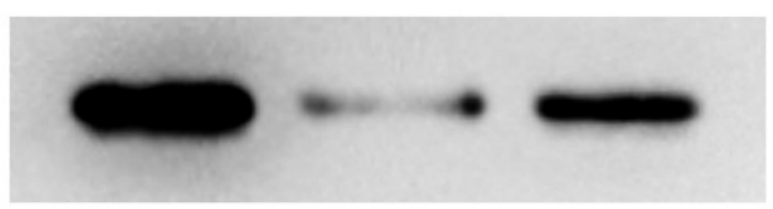

GAPDH

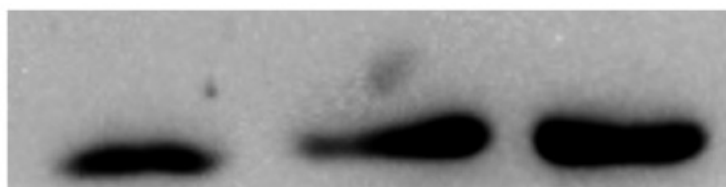

